\title{
The chips are down for Helicobacter pylori
}

\author{
J C Atherton
}

Microarrays can be used to demonstrate differences in genetic content between Helicobacter pylori strains, giving a foretaste of how research may be conducted in the near future

$\mathrm{T}$ he whole genome sequence of Helicobacter pylori strain 26695 was published in 1997 and that of a second strain, J99, in 1999. ${ }^{2}$ These publications moved $H$ pylori research forward by a quantum leap. Comparing $H$ pylori genes with genes of known function in other bacteria gave immediate insights into $H$ pylori metabolism, structure, adaptive mechanisms, and virulence. Research became more focused in that workers could identify and study genes that were known to be important in other bacteria and appreciate their whole genetic context in $H$ pylori. For example, they could immediately see where an enzyme might fit in a metabolic pathway or what proteins were likely to comprise the $H$ pylori flagella assembly. Now, several groups have taken another quantum leap forward. They have constructed whole genome microarraysordered arrays of all identified $H$ pylori genes on glass slides-allowing experiments to be performed on all $H$ pylori genes simultaneously. Such microarrays have already been used to assess differences between $H$ pylori strains ${ }^{3}$ but the paper by Israel et al shows how they can be applied to research into pathogenesis.

Israel et al took two strains of $H$ pylori, one from a patient with a duodenal ulcer (DU) and one from a patient with a gastric ulcer (GU), and used them to infect Mongolian gerbils. The $H$ pylori infected Mongolian gerbil is the best (but still an imperfect) animal model of human $H$ pylori infection. Initial strain typing by Israel et al had suggested that both strains were fully virulent in that they both possessed several of the well known virulence markers, including the gene cagA (cytotoxin associated gene A). However, in the gerbil infections, the strain from the GU patient caused a much more severe gastritis and in a few gerbils caused gastric ulcers and gastric atrophy. In vitro, the GU strain stimulated epithelial cells to produce high levels of the proinflammatory cytokine interleukin 8 . In contrast, the DU strain caused only mild gastritis in gerbils with none in the gastric corpus and stimulated only low level interleukin 8 production by epithelial cells in vitro. The DU strain also caused less epithelial cell proliferation and apoptosis than the GU strain, both in the animal model and in vitro.

The next step was to assess differences between the DU and GU strains of $H$ pylori, and to do this Israel et al used

Israel DA, Salama N, Arnold CN, et al. Helicobacter pylori strain-specific differences in genetic content, identified by microarray, influence host inflammatory responses. J Clin Invest 2001;107:61 1-20.

Helicobacter pylori enhances the risk for ulcer disease and gastric cancer, yet only a minority of $H$. pylori-colonized individuals develop disease. We examined the ability of two $\mathrm{H}$. pylori isolates to induce differential host responses in vivo or in vitro, and then used an $H$. pylori whole genome microarray to identify bacterial determinants related to pathogenesis. Gastric ulcer strain B128 induced more severe gastritis, proliferation, and apoptosis in gerbil mucosa than did duodenal ulcer strain G1.1, and gastric ulceration and atrophy occurred only in B128+ gerbils. In vitro, gerbil-passaged B128 derivatives significantly increased IL-8 secretion and apoptosis compared with G1.1 strains. DNA hybridization to the microarray identified several strain-specific differences in gene composition including a large deletion of the cag pathogenicity island in strain G1.1. Partial and complete disruption of the cag island in strain B 128 attenuated induction of IL-8 in vitro and significantly decreased gastric inflammation in vivo. These results indicate that the ability of $H$. pylori to regulate epithelial cell responses related to inflammation depends on the presence of an intact cag pathogenicity island. Use of an $\mathrm{H}$ pylori whole genome microarray is an effective method to identify differences in gene content between $H$. pylori strains that induce distinct pathological outcomes in a rodent model of $H$. pylori infection.

microarrays. They hybridised DNA from their two strains with copies of genes from the two genome sequenced strains, 26695 and J99. Hence they were able to show which genes were common to the GU and DU strains and which were different. The most marked difference was that the GU strain lacked a run of genes in the cag pathogenicity island. The cag pathogenicity island comprises a collection of genes next to $\operatorname{cag} A$ which have previously been shown to be important in inducing epithelial cells to produce proinflammatory cytokines (such as interleukin 8 ) and thus cause inflammation. ${ }^{45}$ Israel et al went on to experimentally mutate the GU strain by inactivating and deleting its cag pathogenicity island. They infected gerbils with the GU strain and its cag $^{-}$mutants. The cag mutants of the GU strain behaved very like the DU strain in that they produced less inflammation than the wild-type GU strain in the antrum and virtually none in the corpus. They also behaved like the DU strain in vitro stimulating only low level interleukin 8 production from epithelial cells. However, there were slight differences from the DU strain, the most obvious of which was that the cag mutants still induced a similar level of epithelial cell apoptosis to their wild-type GU parent strain. Taken together, this work implied that most of the differences between the
DU and GU strains, and in particular the severity and distribution of gastric inflammation, were due to disruption of the cag pathogenicity island in the original DU strain. However, some differences, such as the effect on apoptosis, were due to other differences between the strains.

This study has several important messages. It confirms and extends a recent report using a cagE mutant (cagE being an important component of the cag pathogenicity island) which showed that functional cag pathogenicity island genes are important in $H$ pylori induced gastric inflammation in the gerbil. ${ }^{6}$ Importantly, it adds clinical relevance to this finding by showing that a strain with a natural partial cag deletion has a similar effect. However, it does not help us to understand differences between $H$ pylori strains associated with GUs or DUs as only single candidate strains were studied. Indeed, the DU strain in this study is atypical in that most DU strains have an intact cag pathogenicity island like the GU strain studied here. ${ }^{7}$ The findings in this paper are important but perhaps the study's best aspect is that in using microarrays to demonstrate strain differences throughout the genome, it gives a foretaste of how research may be conducted in the near future. What is more, as the paper's discussion points out, whole genome microarrays will get better. The microarray used in this study 
could only detect major differences in genetic content whereas in the near future microarrays will be able to detect small differences in individual genes. That will be the next leap forward.

\section{ACKNOWLEDGEMENTS}

John Atherton is funded by a Senior Clinical Fellowship from the Medical Research Council.

Gut 2002;50:293-294

\section{Author's affiliation}

J C Atherton, Division of Gastroenterology and Institute of Infections and Immunity, University

Hospital, Nottingham NG7 2UH, UK

John.Atherton@nottingham.ac.uk

\section{REFERENCES}

1 Tomb JF, White O, Kerlavage AR, et al. The complete genome sequence of the gastric pathogen Helicobacter pylori. Nature 1997:388:539-47.

2 Alm RA, Ling LS, Moir DT, et al. Genomic-sequence comparison of two unrelated isolates of the human gastric pathogen Helicobacter pylori. Nature 1999:397: 176-80.

3 Salama N, Guillemin K, McDaniel TK, et al. A whole genome microarray reveals genetic diversity among Helicobacter pylori strains. Proc Natl Acad Sci USA 2000;97:1466873.

4 Tummuru MK, Sharma SA, Blaser M. Helicobacter pylori pic $B$, a homologue of the Bordetella pertussis toxin secretion protein, is required for induction of IL-8 in gastric epithelial cells. Mol Microbiol 1995; 18:867-76.
5 Censini $S$, Lange $C$, Xiang $Z$, et al cag, a pathogenicity island of Helicobacter pylori, encodes type l-specific and disease-associated virulence factors. Proc Natl Acad Sci USA 1996:93:14648-53.

6 Ogura K, Maeda S, Nakao M, et al. Virulence factors of Helicobacter pylori responsible for gastric diseases in Mongolian gerbil. J Exp Med 2000;192:1601-10.

7 Jenks PJ, Megraud F, Labigne A. Clinical outcome after infection with Helicobacter pylori does not appear to be reliably predicted by the presence of any of the genes of the cag pathogenicity island. Gut 1998;43:752-8.

8 Kidd $M$, Lastovica AJ, Atherton JC, et al. Conservation of the cag pathogenicity island is associated with vacA alleles and gastroduodenal disease in South African Helicobacter pylori isolates. Gut 2001:49:11-17.

\section{7th European Forum on Quality Improvement in Health Care}

We are delighted to announce this forthcoming conference to be held in Edinburgh, Scotland on 21-23 March 2002. Delegate enquiries are welcome.

The themes of the Forum are:

- Leadership, culture change, and change management

- Achieving radical improvement by redesigning care

- Health policy for lasting improvement in health care systems

- Patient safety

- Measurement for improvement, learning, and accountability

- Partnership with patients

- Professional quality: the foundation for improvement

- Continuous improvement in education and training

- People and improvement.

Presented to you by the BM Publishing Group (London, UK) and Institute for Healthcare Improvement (Boston, USA). For more information contact: quality@bma.org.uk or look at the website www.quality.bmipg.com. Tel: +44 (0)20 7383 6409; fax: +44 (0)20 7373 6869. 